sallow complexion, applied to me for advice. She stated that she had long felt very weak and poorly, and attributed the change in her health to an attack of bowel complaint, which she experienced the previous autumn. Since then she had lost strength and flesh, had had no appetite, and had been generally unwell. She had also suffered from whites and from a pain in the lower part of the back, the result of weakness, she considered. The above account of her symptoms, coupled with the expression of the physiognomy, inducing me to suspect the existence of uterine inflammation, I at once directed my inquiries to the state, past and present, of the uterine system. It appeared that, although of delicate constitution, she had always enjoyed tolerable health up to within the last five or six months. Menstruated at fourteen; she continued to do so regularly and easily both until and after her marriage, which occurred at the age of four-and-twenty. She has since had two children. The last was born two years ago. The labour was rather tedious, but natural, and she soon recovered. She nursed her child during seven months, when it was weaned. During that time she does not recollect having experienced any uterine pain, or having suffered from leucorrhoal discharge. The catamenia returned, as usual, on her ceasing to nurse, and have continued to make their appearance regularly until the present epoch. From the time that she had the bowel complaint last autumn, however, they have been painful, scanty, and have lasted six days instead of four. Since that epoch, also, she has had a slight leucorrhoeal discharge. On questioning her minutely, $I$ also found that it was more especially since then that she had experienced the pain in the back; and that she had also had pain low down in the hypogastric region, although her attention had not been directed to the circumstance. She stated likewise, on inquiry, that she experienced pelvic weight and heaviness after any fatigue, or after standing long.

The presence of these symptoms necessitating further scrutiny, I examined digitally, and found the cervix rather low in the vagina, voluminous and retroverted, but soft, or, at least, elastic. The lips of the os nteri were widely open, so as to admit the extremity of the medius, and presented the peculiar soft velvety sensation of ulceration. No great sensibility on pressure. The uterus appeared small and healthy. As there was evidently inflammation and ulceration of the cervix, I told her to procure a letter for the Western Dispensary.

On examining her there on the 10th, with the speculum, I found, as I had anticipated, that the os uteri was ulcerated; the ulceration extending on both lips, and into the open os, so as to present, externally, a surface a little larger than a shilling. The ulceration was not unhealthy, the granulations being small and florid; the cervix itself was greatly enlarged, but not indurated, the hypertrophy being evidently inflammatory. The mucous membrane of the cervix, and of the upper part of the vagina, was of a deep-red hue, and covered with thick yellow muco-pus. Tongue rather white; appetite bad; bowels costive.

Treatment.-To take a mild, saline, opening mixture daily; to use a sulphate-of-zinc injection twice a day. Periodical cauterization of the ulceration with the pernitrate of mercury, or the nitrate of silver. Rest in the horizontal posture as much as possible. Mild diet; no stimulants.

March 1st. - The leucorrhoal discharge is much less abundant, and less purulent; the cervix, which is higher in the vagina, is still large to the touch, although less than it was at first; the vagina and cervix are less injected; the ulceration on the cervix appears healed at first; but on expanding the bivalve speculum, it is found still to exist in the cavity of the os, which is large enough to admit the extremity of the middle finger; the pain in the back is much less; there is scarcely any in the hypogastric or inguinal regions; the general health is much ameliorated; the appetite and digestive functions are improved; the bowels are less confined, and the rest is better. The periodical cauterization and astringent injections to be continued. Citrate of iron to be taken internally, instead of the saline mixture.

14th.-The local and general improvement continues; the menses have just appeared, and are easier and more abundant than they have been for many months.

April 1st.- The ulceration is healed externally, and in all that part of the cavity of the cervix which the eye can reach, when the lips of the os uteri are fully expanded by the bivalve speculum; but there are still a few drops of muco-pus issuing from the os, and on introducing the extremity of the uterine probe into the os, its contact is followed by the effusion of a drop or two of blood. The ulcer evidently still existing in the interior of the cavity of the cervix, a pointed stick of nitrate of silver was pushed into the os for nearly half an inch.

This internal cauterization of the cervix was resorted to three or four times, at the interval of a week, along with coldwater vaginal injections, with complete success. On the 11th of April, every trace of inflammation and ulceration of the cervix had disappeared, inside of the cavity of the os as well as outside; the cervix is still rather voluminous and retroverted, but soft in its entire extent, and high up in the vagina; the mucous membrane of the cervix is rather more rosy than usual, and there is on it a scanty white secretion; she has no pain in the hypogastrium, nor in the inguinal regions; she has neither any pain in the back, nor any sensation of bearing-down, except after fatigue, when she still feels slightly the two latter symptoms; the tongue is quite clean; the appetite and rest good; she is very much stronger-indeed, quite well I recommended her to continue the cold-water injections, to avoid fatigue, and to be careful as to diet.

In the middle of May, I again examined this patient, as a precautionary measure, and found that the cicatrization had remained perfect; and there was complete absence of local inflammation or irritation. She was evidently quite well and safe from relapse.

Cambridge-square, Hyde-park, June, 1846.

$\overline{\overline{\text { The }}}$

\section{USE OF THE STARCH BANDAGE IN VARIOUS SURGICAL DISEASES.}

By AlFred Markwick, Esq., M.R.C.S. London.

IN a paper lately published in The LANCET, (p. 240,) I called the attention of its readers to the use of the starch bandage in the treatment of fractures, and attempted to prove that its advantages were due to the great solidity and support it gave to the fractured $\operatorname{limb}$; to its preventing the displacement of the bones; to the facility with which it man be split open, for the purpose of examining the state of the injured member, and applying such remedies as the case may require; and though last, not least in importance-to its enabling the patient to leave his bed, and move about from place to place, and attend to his accustomed avocations, without either risk or danger, in the majority of cases : his strength being by this means kept up, while those cachectic and debilitated states of the constitution consequent on a prolonged decubitus are prevented.

In the present communication, I propose adverting to its application in those cases in which, as in fractures, the chief indication is to keep the part motionless. These are-dislocations, sprains, and other injuries of the joints; diseases of these parts; ruptures of the muscles and their tendons : re-sections of bones ; necrosis and caries; certain deformities, either congenital or acquired, or from vicious cicatrization aneurisms ; varicose veins; hernia; indurated testicle, \&c. I shall consider each of these in the order in which they are here given.

It is not my intention to enter into a full description of every species of luxation : I shall confine my remarks to the subject of treatment, and more particularly to that portion of it which more directly concerns us in this paper.

There are some dislocations in which it is almost impossible to prevent a repetition of the displacement by the ordinary means-as, for instance, in the dislocation, forwards, of the sternal end of the clavicle. Now with the starch bandage we can effectually overcome this difficulty.

The indications in this accident are, to keep the shoulder outwards and forwards, and the sternal end of the clavicle in its proper situation. The best apparatus for fulfilling these indications is a starch bandage, consisting of a combination of a portion of Dessault's bandage for fractured clavicle and the anterior figure-of- 8 bandage. The former, which should only be sufficiently starched to prevent it from getting slack, will keep the shoulder outwards by means of the axillary pad, while the latter will bring it forwards and keep the sternal end of the bone in its place by its firmness and solidity-properties that are due to the starch, with which it should be abundantly covered, especially over the sterno-clavicular articulation. Should more firmness be required to effect this object, a piece of paste-board or stiff leather, previously soaked in warm water, and starched, may be applied, and secured by a second figure-of- 8 bandage. The arm is then to be supported in a sling. As the axillary pad, by pressing on the vessels of the arm, has a tendency to produce œdema, it is always advisable to commence by passing a roller around the limb from the fingers upwards. 
This example will, I think, sufficiently show the importance of the starch bandage in the treatment of luxations. ${ }^{*}$ I may, however, state, that it does not, as in fractures, constitute a distinct apparatus; on the contrary, the contentive means and mode of treatment, in each particular case, remain the same, the only difference being in the starch with which the bandage is covered, for the purpose of increasing its solidity and streng th, and preventing it from becoming loose.

Sprains and other injuries of the joints constitute the next class of cases. When called to a case of sprain immediately after it has happened, the first thing to be done is to elevate the limb, and place it in the most easy and comfortable position for the patient, and then to adopt such measures as are calculated to prevent, if possible, the occurrence, or, at all events, to check the violence, of the inflammatory action. The immediate application of cold, and persevered in for a sufficient length of time, seems to be the most effectual means of preventing the aftlux of the fluids towards the part, upon which the inflammation depends. When the inflammatory period has passed, no time should be lost in placing the joint in a starch bandage, which is to be applied in the manner directed in the first paper, with or without the pasteboard splints, as the case may be. This apparatus, by accurately moulding itself on all the inequalities of the articulation, forms for it a continuous, permanent, and immovable splint, which not only keeps it pertectly free from all motion, but likewise gives it that support by which the patient is enabled to get about much sooner, and with far greater safety, than he, by possibility, can do when a movable apparatus is employed. If, instead of being sent for immediately after the accident, as I have supposed to be the case in the foregoing paragraph, we do not see the patient until some time afterwards, when there is considerable tumefaction and ecchymosis, the same precautions are necessary with respect to the perfect quietude of the joint but the employment of cold, which was so beneficial in the preceding instance, is here more injurious than useful. Recourse should, in these cases, immediately be had to either general or local bloodletting, or both, to the extent required by the severity of the injury, and the size of the joint affected, followed by warm, emollient, sedative fomentations and poultices; and then, when the inflammation has been subdued by these means, the application of the starch bandage should be forthwith proceeded with. If the case has become chronic, and there is effusion of serum into the synovial membrane, together with considerable stiffness and weakness of the articulation, then the remedies recommended as applicable to the preceding stages must be replaced by others of a stimulating character, such as friction with camphorated and ammoniated liniments, blisters, \&c., with a view to promote the absorption of the effused fluid, and the joint placed as quickly as possible in a starched bandage, which will, in the majority of cases, be found the most powerful and effectual resolutive means. . In this stage, the ligaments are considerably relaxed and weakened, and, in order to regain their strength and firmness, require to be kept perfectly quiet, and well supported. Nothing can be better suited for this purpose than the starch bandage, from the uniform pressure it produces, and the solidity and immobility it possesses.

Pulpy thickening of the synovial membrane.-From the nature of this affection, it is evident that perfect rest must constitute the only means upon which we can at all calculate for producing any benefit. Mr. Scott employs for this purpose strips of plaster, but they are not sufficiently efficacions, and, moreover, are not free from disadvantages. One of these, is their great tendency to produce excoriation, and hence to necessitate their frequent removal; and another, if possible, still greater, is, that when abscesses are present, they prevent the free escape of the matter, and become filthy and offensive in consequence. The starch bandage is an admirable remedy in these cases, as it can be so applied as both to produce the effect desired, and allow of a free discharge of all purulent matter, and, at the same time, to check its further accumulation.

Ulceration of the cartilages.-As ulceration cannot be put a stop to, but, on the contrary, is aggravated by friction, it is clear that the only means by which we can arrest or check its progress is to keep the joint in the most perfect state of im-

* Since writing the above, I have been much interested in reading in ThE LANCET for March 14th, a report of a case of " Remarkable Disease of the Ankle-joint," related by Mr. Harding to the Westminster Medical Society, in which a marked good effect had resulted trom keeping the joint perfectly motionless by means of an apparatus, formed after the manner of Mr. Smee's moulding-tablets, of a roller saturated with a mixture made by rubbing together prepared chalk and gum mucilage. I need not say that the starch bandage would have produced equally good results, and with less trouble. mobility. This constitutes the most important part of the reatment, and must not be neglected. By adopting this course, we sometimes succeed in entirely curing the disease, provided we are called upon to treat it at a sufficiently early period. But if our advice has not been sought until the process of destruction has farther advanced, and caries has, in all probability, commenced in the heads of the bones, then we have but little, if any, chance of effecting a perfect cure; and we must endeavour, by every possible means, to stop the further progress of the disease, so as to bring about ankylosis. The starch bandage is the best apparatus that $I$ know of for restoring the joint in the first stage, and for arresting the ulceration, and securing the termination by ankylosis in the second. It may be applied either entirely round the joint, or openings may be left in it, for the purpose of applying such remedies as the nature of the case may require, or for the escape of the matter from the various sinuses. Nothing can be more congenial to the patient, or more likely to produce beneficial results, than the moderate but equal pressure which this bandage produces on all parts of the joint. "It will," as Sir B. Brodie says, when speaking of pressure in scrofulous diseases of the joints, "promote the healing of the sinuses, and by more completely preventing the motion of the joint, will lessen the chance of fresh suppuration, and favour the union of the ulcerated bony surfaces."

White swelling.*-This disease has its origin in the cancellated structure of the bones, consequently is generally met with in those situations in which this tissue is the most abundant-viz., in the knee and elbow joints, and in the small bones composing the tarsus and carpus. The tarsus and the knee are the most frequently affected.

The treatment in this affection is much the same as that required by the last-mentioned disease, the indications at the commencement being, to arrest the progress of the ulceration and prevent the other structures from becoming affected; and, at a more advanced period, when these have become diseased, and abscesses have formed, to endeavour to save the limb by promoting ankylosis. Absolute repose, therefore, of the affected joint is of the utmost necessity, as the slightest motion irritates the diseased bones, accelerates the ulceration in them, and hastens its extension to the adjoining parts. The starch bandage will prove an invaluable apparatus to the surgeon in these cases. It readily admits of the application of external remedies, such as issues, blisters, or other counterirritants, and also of the free exit of the discharge produced by these, or resulting from the abscesses that may have formed; while at the same time it gives to the joint the necessary support, and prevents all motion between the articulating surfaces of the bones. In cases where large abscesses have formed, it will be found of great service, by the uniform pressure which it produces, in dispersing the purulent matter which they contain, and in suppressing its further secretion, and by this means bringing the parts into the condition necessary for the production of ankylosis.

For the therapeutic treatment of the diseases of the joints, I must refer to the various surgical works, and especially to Sir B. Brodie's elaborate treatise, in which it is fully described.

Rupture of the muscles and tendons.-As the perfect restoration of the use of the limb will depend on the close approximation of the lacerated parts, it follows that in the treatment of these injuries, the member must be placed in such a position as will perfectly relax the ruptured muscle or tendon, and bring its severed extremities in close apposition, and a suitable apparatus must be employed to maintain them in this condition. The starch bandage will be found the most efficacious one for this purpose. It retains the limb in the requisite state for the perfect coaptation of the ruptured surfaces, and prevents the contraction of the muscles, upon which a separation frequently depends.

Let us take, by way of illustrating its advantages, one of the most serious of this class of accidents-viz., a case of rupture of the tendon of the rectus femoris muscle. In this, there is generally considerable subsequent weakness and lameness in the $\operatorname{limb}$, owing to the inability of the ordinary remedies to keep the parts in a necessary state of extension. Now if a starch bandage be employed, it will overcome every difficulty, and fulfil every indication. During its application the limb must be completely extended, and the coaptation made, by depressing the upper portion of the muscle, and raising the

* White-swelling is a term that has been applied by various authors to very different diseases, such as inflammation of the synuvial membrane, pulpy thickening of the same, ulceration of the cartilages, and caries of the
heads of bones. It is, however, to the last that it is the most applicable, from the circumstance that the colour of the skin remains the same. 
patella by means of graduated compresses. The starch bandage will also be found of great service in cases of spasmodic affections of the muscles, as in chorea, \&c.

$R e$-section of the heads of bones. - This operation is had recourse to when we wish to remove the disease in them without sacrificing the limb. It must therefore be performed before the surrounding soft structures become implicated, and before the patient's health is seriously affected. After the operation, when the wound has nearly or quite healed, the joint requires to be confined in a certain position, and kept perfectly quiet for some time, during the formation of the fibrous tissue, by which the bones eventually become united. The starch bandage in these cases is a very useful apparatus.

In caries and necrosis of the bones also, and in the inflammation which precedes them, the firm and equable pressure which this bandage produces will be of great service in checking the accumulation of matter, and in securing the perfect repose of the limb, by which means a considerable degree of irritation will be prevented.

Congenital Deformities.-The first of these that I shall mention is spina bifida. The treatment consists in evacuating the fluid of the spinal tumour, and then replacing and maintaining the protruded membranes within the vertebral cavity. The advantage of pressure and puncture in these cases was fully exemplified by the success the late Sir Astley Cooper obtained from it in two instances.*

Although I have not had an opportunity of witnessing the effects of the starch bandage in the affection under consideration, I can but think, that if properly applied, and care is taken to protect the integuments, covering the tumour with some soft material, in order to prevent inflammation and excoriation, it would prove an exceedingly useful and effectual apparatus. It certainly recommends itself for trial.

Another frequently congenital deformity is club-foot, of which there are three varieties. In these cases, the object in the treatment is to overcome the inordinate contraction of the mnscles, by which the different varieties are produced. This can be effected, in many instances, when the child is not too old, by apparatus, which both restrain the further action of the muscles, and tend forcibly to bring the foot into its normal position. In some cases it is necessary previously to divide the tendons. Most of the mechanical contrivances that are employed for this purpose are costly, and consequently beyond the reach of the poorer classes. In the starch bandage we have a cheap and convenient remedy, one equally efficacious, and therefore equally, if not more valuable.

There are certain other non-congenital deformities, produced either by the permanent contraction of the muscles or by the shortening and rigidity of the fasciæ, or by the gradual contraction of the cicatrices, resulting from burns or extensive ulceration, for which the starch bandage will be equally applicable, after an operation has been performed, for the purpose of overcoming either the contraction of the muscles or of the cicatrices, or counteracting the gradual shortening of the fasciæ. To this class belong contracted fingers and various kinds of spurious ankylosis, as of the knee and elbow joints.

The deformities arising from burns are frequently very considerable, and often perfectly irremediable. Thus the bones have been known to be dislocated, the joints firmly flexed or bent backwards, the head drawn on one side, the chin united to the integuments covering the sternum, and the thigh to the abdomen. It is always advisable to prevent these sad results as much as possible, by the application of bandages during the process of cicatrization, so as to keep up a constant extension in the opposite direction to that in which the deformity is about to be produced. I know of no apparatus that will be found so effectually to attain this object, and with so little inconvenience to the patient or the practitioner, as the bandage under consideration. It may be applied over the ordinary dressings.

In aneurisms and varicose veins it is extremely useful. In the former, its even, but firm pressure, equalizes the circulation through the $\operatorname{limb}$, and by lessening the impetus with which the blood is sent into the aneurismal sac, prevents its dilatation, and promotes the coagulation of its contents, and its subsequent obliteration. In the latter, the support it gives to the limb prevents any undue accumulation of blood in it, and enables the dilated and distended veins to contract on their contents, and propel the blood onwards towards the heart, while its firm and unyielding nature effectually secures them from all external injury.

In umbilical and ventral hernia there is no more certain

* For a detailed account of these cases, see the second vol. of the "Medico-Chirurgical Transactions," and Cooper's "Dictionary," article spina bifida. means of preventing the protrusion of the bowel than the starch bandage. It is applied in the following manner:-The little patient being suspended in the air, in the horizontal position, by two assistants, the surgeon proceeds to return the intestine into the cavity of the abdomen, and having done so, places over the hernial aperture the apex of a graduated compress, upon which firm pressure is made by an assistant. $\mathrm{He}$ then takes up a fold of the integuments on each side of the graduated pad, while another assistant passes round the body a linen band, about six or seven inches wide. The whole is then firmly secured by a well-starched roller.

The application of this bandage may be extended to other herniæ, both in children and in adults.

In indurated testicle its advantages are very apparent. It produces much more firm and equal pressure than any strapping can do, and does not cause that painful excoriation of the skin which this does invariably.

I might mention several other cases in which it would be beneficial; but $I$ have already given sufficient examples to show its value and importance.

Langham-place, April, 1846.

\section{REMARKABLE CASE OF ABSCESS OF THE} HEART.

PAIN IN THE LEG THE ONLY SYMPTOM OF DISEASE DURING LIFE.

By T. How ITt, Esq., Surgeon to the Lancaster Infirmary.

Observing in The Lancet for May 16th last, (p. 548, the history of a "rare case of abscess of the heart," by Mr. Chance, I am induced to send the particulars of the subjoined case I have transcribed it just as it was entered in my note-book at the period it occurred. I still possess the morbid specimen; and as it appears from Professor Owen's statement to $\mathbf{M r}$. Chance, that there is not one similar in the museum of the College of Surgeons, I purpose to deposit it there.

On November 18th, 1833 , at eight P.M., I was requested by my lamented friend, Mr. John Merriman (then house-surgeon to the Lancaster Infirmary) to visit Samuel $P \longrightarrow$, eight years of age. I found him suffering from most acute pain, which he described as deeply situated towards the centre of the calf of the right leg, having commenced suddenly about twelve hours previously. So far as we could learn, it had not been produced by any external agent, he having had neither blow nor fall. Upon a careful examination of the part, we could detect neither swelling nor redness, nor any symptom indicative of inflammation, neither was there any spasmodic action of the muscles to account for it. Occasionally the pain remitted in severity. When he complained of violent throbbing; our examination did not appear to cause any increased pain; his bowels had been relieved by a dose of castor-oil exhibited by Mr. Merriman this morning; no headach, no pain in the chest or abdomen, no thirst, pulse 110 ; - in fact, this pain in the leg was the only complaint the boy had to make.

Supposing, from the history, that matter might be about to form under the periosteum, we direeted six leeches to be applied over the seat of the pain, and small doses of calomel and opium every four hours.

19th.-Nine A.M. : No relief; the pain as acute as yesterday, yet no swelling or redness, except around the leech-bites which had bled pretty freely. Having during the night voided two large lumbrici, he was ordered a turpentine injection, and the calomel and opium to be continued; pulse 120 .

20th.-Nine A.M. : The pain in the leg still contimues; his general condition is much the same, but he appears a little dull and stupid, not answering questions very readily, though quite correctly; pulse 130, more feeble. The mouth not being at all affected by the calomel, we imagined the dulness he evinced to be the effect of the opium, and mercury with chalk, oombined with rhubarb, was substituted for the calomel and opium. The bowels had been twice relieved by the turpentine enema, and three more lumbrici voided.

21 st.- Nine A.M. : No mitigation of the pain in the leg, nor any further evidence as to its cause; the limb preserves its natural heat and size. I directed it to be well rubbed with hot turpentine, and then enveloped in a warm poultice. In other respects, little variation from yesterday. Pulse 130 feeble.- Eight P.M. : Decided symptoms of coma now making their appearance. Pulse 140; pupils contracted; the patient lying upon his back, constantly moaning; with difficulty roused, but when roused, quite sensible, and still complaining of his leg. Ordered a small blister to the nape, and a teaspoonful of wine to be given occasionally. Bowels relieved by an enema.

22nd.-Nine A.M. : Rallied a little; less stupor ; perfectly: 